\title{
Can we use medical examiners' records for suicide surveillance and prevention research in Nova Scotia?
}

\author{
L. A. Campbell, MSc (1,2,7); L. Jackson, PhD (3,4); R. Bassett, PhD (5); M. J. Bowes, MD (6); M. Donahue, MDiv, MAHSR(c) (7);
} J. Cartwright, MA (2); S. Kisely, MD, PhD $(1,2,8)$

This article has been peer reviewed.

\begin{abstract}
Introduction: Medical examiners' records can contribute to our understanding of the extent of suicide in a population, as well as associated sociodemographic and other factors.

Methods: Using a mixed methods approach, the key objective of this pilot study was to determine the sources and types of information found in the Nova Scotia Medical Examiner Service (NSMES) records that might inform suicide surveillance and targeted prevention efforts. A secondary objective was to describe the one-year cohort of 108 individuals who died by suicide in 2006 in terms of available sociodemographic information and health care use in the year prior to death.
\end{abstract}

Results: Data extraction revealed inconsistencies both across and within files in terms of the types and amounts of sociodemographic and other data collected, preventing correlational analyses. However, linkage of the records to administrative databases revealed frequent health care use in the month prior to death.

Conclusion: The introduction of systematic data collection to NSMES investigations may yield a comprehensive dataset useful for policy development and population level research.

Keywords: suicide, population surveillance, medical examiner, coroner, administrative data

\section{Introduction}

With approximately 90 recorded deaths due to suicide in Nova Scotia each year, suicide is a considerable public health problem, despite being largely preventable. ${ }^{1}$ In addition to being highly traumatic for family members and friends, suicide is costly. The potential years of life lost (PYLL) due to suicide are substantial: for those aged under 74 years, only cancers (all sites), circulatory disease and unintentional injuries accounted for more PYLL from 2005 to 2007 . $^{2}$ These figures may well be underestimates, since suicide is widely believed to be underreported.

A number of factors contribute to the under-reporting of suicides, such as failing to suspect suicide (particularly among the elderly or in the absence of notes or other indications of a possible suicide). In addition, determining intent is particularly difficult in some instances, such as in deaths due to poisoning. Rates of suicide by poisoning may be underestimated by approximately $30 \%$, relating to a $10 \%$ underreporting of overall suicide rates. ${ }^{3}$ An Ontario study of the validity of death certification of unnatural adult deaths highlighted the difficulty in determining intent due to the subjectivity of interpretation. ${ }^{4}$ Deaths due to hanging or inhalation of noxious gas were more likely to be attributed to suicide than those due to poisoning or drowning; death due to overdose of overthe-counter medication was certified more frequently as suicide than death as a result of heroin overdose. Increasing proof of intent resulted in increased odds of correct certification as suicide. In addition, some physicians may be reluctant to report suicide as the cause of death due to stigma or financial implications for family members. ${ }^{5}$

When suicide is suspected, the manner of death is determined in a medico-legal process that can be informed by different types of evidence, including an investigation of the scene, post-mortem examination results, collection of medical histories and circumstantial information. The systems for the investigation of suicides vary across Canadian provinces and territories; some jurisdictions possess a medical examiner system and others a coroner system. ${ }^{6}$ Medical examiners are physicians, while coroners may have legal,

Author references

1. Department of Psychiatry, Dalhousie University, Halifax, Nova Scotia, Canada

2. Department of Community Health \& Epidemiology, Dalhousie University, Halifax, Nova Scotia, Canada

3. School of Health and Human Performance, Dalhousie University, Halifax, Nova Scotia, Canada

4. Atlantic Health Promotion Research Centre Dalhousie University, Halifax, Nova Scotia, Canada

5. Faculty of Health Professions, Dalhousie University, Halifax, Nova Scotia, Canada

6. Nova Scotia Medical Examiner Service, Halifax, Nova Scotia, Canada

7. Capital District Health Authority, Halifax, Nova Scotia, Canada

8. Health LinQ, University of Queensland, Queensland, Australia

Correspondence: Leslie Anne Campbell, Room 230, Centre for Clinical Research, 5790 University Avenue, Halifax NS B3H 1V7 Tel.: (902) 473-7458; Fax: (902) 473-4546;

Email: leslie.anne.campbell@dal.ca 
investigative or medical backgrounds. In 1960, the Fatality Inquiries Act established the Nova Scotia Medical Examiner Service (NSMES); a 1989 amendment to the Act established a provincial Chief Medical Examiner (CME). ${ }^{6}$ The current iteration of the provincial medical examiner system operates out of a central office in Halifax, Nova Scotia.

NSMES is responsible for investigating "all deaths due to violence, undue means, culpable negligence and unexpected/unexplained deaths throughout the province," ${ }^{\text {" }}$ which includes all deaths due to suicide. The primary role of NSMES is to identify the decedent; establish the date, time, place and cause of death; and, in the case of apparent suicide, determine the intent. These duties are described in detail in the Nova Scotia Fatality Investigations Act. ${ }^{7}$ The scope of each investigation varies depending upon the circumstances of death, but the aim is always to determine intent.

Clearly, accurately classifying suicide is necessary to identify those factors that may serve as target points for intervention and prevention strategies. However, a lack of standardized criteria for classifying suicide and difficulties in applying these criteria in a consistent fashion contribute to potential inaccuracies in classification. ${ }^{4,8,9}$

Despite these limitations, medical examiners' records are important sources of information and may contribute to our understanding of both the extent of suicide in a population and associated sociodemographic and other factors. ${ }^{9-14}$ Similar records have proven useful for research and surveillance in other jurisdictions, including elsewhere in Canada, England and the United States. ${ }^{11,13,15-20}$ However, information collected by NSMES to date has not been used for surveillance, and only on occasion for research. ${ }^{21}$

Research suggests several individual risk factors associated with suicide: many decedents have a history of mental health or addiction problems $\mathrm{s}^{22,23,24}$ and men and boys appear to be at elevated risk, often through the use of more lethal methods. ${ }^{5,15,20,25,26}$ Other reported risk factors include increasing age ${ }^{26}$ rural residence, ${ }^{18}$ household firearm ownership, ${ }^{27}$ social isolation, ${ }^{25}$ low socio-economic status, ${ }^{18,26}$ chronic pain, terminal illnesses or disabilities, ${ }^{28}$ or being the victim or perpetrator of domestic violence. ${ }^{20}$

NSMES investigations provide an opportunity to collect more detailed information, including on known risk factors. Further, medical examiner and other death investigation systems have specific geographical mandates, creating population-based data sources. Death certificates or trauma registries contain incomplete information about deaths due to suicide, and as such cannot alone inform prevention policies or epidemiological research.

The purpose of our research was to examine the content of the information collected by NSMES for suicide cases to: (1) determine the types and sources of available information that might be useful for suicide prevention research; and (2) develop a "profile" of suicides in order to highlight the information that could be used as part of an ongoing surveillance system. For the latter objective, we linked each suicide to health service data from the provincial administrative databases to provide a profile of health service use in the year prior to the death.

The Dalhousie University Ethics Review Board and the Nova Scotia Department of Justice reviewed and approved the research prior to the collection of any data.

\section{Methods}

We used a mixed methods approach, the qualitative component to assess the types and sources of information available in the files, and the quantitative component to provide a "profile" of suicides in Nova Scotia. For each component, we manually extracted data from NSMES records for all deaths due to suicide for a one-year period from January 1, 2006, to December 31, $2006(n=108)$. We chose a one-year period in order to obtain enough data to effectively assess the types and sources of information in the files and to build a profile of suicides; the year 2006 was the most recent one-year period for which all files on suicide were "closed," that is, no new information would be added to the file.
Qualitative component: types and sources of information

For the qualitative component of the study, our research assistant reviewed each suicide file to record the existence of discrete sociodemographic and other related information (e.g. where the body was found) and ascertain other types of information within the files and the source(s) of this information. For example, information given by a family member concerning the social life of the decedent was recorded as "family member provides social information," with no other identifying information.

Data regarding the types and sources of other information were extracted and recorded in a text document. This text document was then imported into the computer software program NVivo 7 (QSR International) to manage and sort by source and type of information. Our primary qualitative researchers (LJ, RB) regularly provided feedback throughout the extraction and sorting process to ensure that a comprehensive listing of types and sources of information was captured. Memos were developed and constantly updated throughout data extraction to note any modifications to the working definitions of categories of sources and types of information. Modifications were made when a working definition was deemed incomplete.

We developed various categories (e.g. legal issues, social issues) for the different types of information and defined sources as the people (e.g. physicians), places (e.g. morgues) and documents (e.g. health records) that provided information.

\section{Quantitative component: profile of suicides}

The quantitative component of the study consisted of constructing an anonymized Access 2003 (Microsoft) database based on the information extracted manually from the NSMES files. The database included available information collected by the medical examiner service on demographic, personal, social and event-related factors (e.g. cause of death, precipitating circumstances) and prior health service use. Data were exported from the database into an Excel 2003 (Microsoft) spreadsheet and checked for duplicates and errors before being analysed using Statistical Package for Social Sciences v 12 (SPSS). 
Where possible, these data were linked to the provincial health service administrative databases to determine decedents' health service use (inpatient and outpatient general and mental health services) in the final year of life. We used the following datasets held by the Population Health Research Unit (PHRU) in the Department of Community Health and Epidemiology at Dalhousie University: Canadian Institute for Health Information (CIHI) Discharge Abstract Database of hospital admission/separation dates and diagnostic codes; fee-for-service claims by physicians; and Mental Health Outpatient Information System.

Linkage of the databases was made possible by means of encrypted health card numbers in a process approved by the Department of Justice, the Dalhousie University Research Ethics Board and the PHRU Data Access Committee.

We calculated quantitative descriptive statistics (rates, percentages) for individual demographic factors reported in the NSMES files and prior health service use in Nova Scotia over a one-year period. Incomplete data capture precluded analysis of known risk factors.

Due to the need to suppress small cells to protect the anonymity of decedents, we classified health care episodes only as mental health or non-mental health, and reported for the year and the month prior to suicide. Despite this relatively high level of aggregation, we were unable to report the specific types of health care use (i.e. inpatient vs. outpatient, mental health vs. non-mental health) within the week prior to suicide due to small numbers. A sample size or power calculation was not required, as the project involved reporting all cases of suicide in Nova Scotia over the given time period and specific hypotheses were not tested.

\section{Results}

\section{Qualitative component: types and sources of information}

In a population of 913462 in Nova Scotia in 2006,108 deaths were due to suicide. ${ }^{29}$ Each of these deaths had been investigated by NSMES and therefore had a file on record. Each of the 108 files showed the same basic structure: information about all processes and communications related to events from the time the medical examiner was contacted until the file was closed and an official report completed by the Chief Medical Examiner. However, the files varied greatly in terms of details.

All files provided information on age, sex, address of residence, the place where the body was found and cause of death. Other sociodemographic and related information, such as marital and employment status, was recorded to varying degrees and sometimes inconsistently. For example, in one section of the file the decedent might be described as married, but in another as separated.

We identified 16 different types of information (e.g. autopsy information, death scene) from the 108 files (see Table 1). We deemed the frequency of information common if it was in $60 \%$ or more of the files, and less common if in fewer than $60 \%$ of the files.

We also found and classified 10 sources of information. Of these 10 sources, 5 were classified as common sources of information as they were in all or the majority (i.e. $60 \%$ or more) of files: family/friends, health records, medical examiner(s)/investigators, physicians (including military physicians) and police (including military police). Fewer than $60 \%$ of the files contained information from other sources; these included consultants (e.g. neuropathologists), emergency medical responders, funeral homes, morgues and tissue banks.

\section{Quantitative component}

The derived quantitative database included available information from the 108 files of decedents' demographics, place of suicide, disclosed intent, cause of death, prior health care contacts, previous suicide attempts, medical and psychiatric diagnoses, and precipitating circumstances. Basic demographic factors (age, sex, address, cause of death and place of death) were recorded consistently across files.

From this database, we determined that the mean age of decedents was 44.7 years (standard deviation [SD] \pm 13.3 years) and individuals in their forties made up onethird of the cases $(n=36)$ (see Table 2 ). The female to male ratio was $1: 5$, with 18 (16.7\%) decedents female and 90 (83.3\%) male. Just over half the sample lived in rural areas (defined as those areas outside Halifax Regional Municipality), which is similar to the general Nova Scotian population average of $59 \%$ in $2006 .{ }^{29}$ The most common causes of death were hanging $(38.9 \%)$, selfpoisoning $(24.1 \%)$ and firearm injuries (19.4\%). The most common locations were at home $(56.5 \%)$ or in a public place such as a bridge, park, woods or beach $(27.8 \%)$.

\section{TABLE 1}

Types of information from NSMES records of suicide case files ${ }^{\mathrm{a}}$

\begin{tabular}{ll} 
Frequency of information & Type of information \\
\hline Common & Autopsy information \\
(i.e. present in $60 \%$ or more files) & Cause of death \\
& Death circumstances \\
& Death notifications - procedures \\
& Death registration \\
& Death scene \\
& Health information \\
& Immediate prior activities \\
& Medical/police response/activities \\
& Place and details/accounts of body discovery \\
& Sociodemographic information \\
& Sociopersonal information \\
& Critical life incidents \\
& Legal issues \\
Less common & Request for specific records or information (e.g. dental records) \\
(i.e. present in fewer than $60 \%$ of files) & Suicide plans or attempts \\
\hline
\end{tabular}

Abbreviations: NSMES, Nova Scotia Medical Examiner Service.

a All cases of death due to suicide manually extracted from NSMES records from January 1, 2006, to December 31, 2006. 
Differences in cause of death by sex were apparent: the most common forms of suicide among women and girls were self-poisoning (38.9\%) and hanging (33.3\%); among men and boys, these were hanging (40\%), firearm injuries $(23.3 \%)$ and self-poisoning $(21.1 \%)$. All of the decedents who died by firearm injury were male, with two-thirds of these deaths due to long gun (rifle or shotgun) discharge. While not all precipitating circumstances were captured in the NSMES files, all of the 18 cases in which a recent break-up of a relationship was reported involved male decedents.

Aside from basic demographic information, not all files contained the same amount of information. For example, while the age and sex of decedents were in all files, employment status was missing in $43.5 \%$. Most files (69\%) were missing information on known previous suicide attempts, and $28 \%$ lacked information on any psychiatric diagnoses. In the case of deaths by hanging, $98 \%$ were missing information on the ligature source, point and degree of suspension. Similarly, in the case of firearm deaths, $90 \%$ lacked information regarding ownership, license status and storage of the firearm.

We supplemented the health service use data by linking our derived database with the provincial administrative health services databases using encrypted health card numbers. Of those decedents whose health card numbers were retrievable $(\mathrm{n}=101)$, most $(74 \%)$ had been in contact with health services (either as an in- or outpatient) in the year prior to suicide; over half ( $55 \%$ ) had been seen as outpatients and nearly one-quarter (23\%) had been hospitalized for mental health reasons in the year preceding suicide; $10 \%$ had been in hospital for mental health reasons in the month prior to suicide; $16 \%$ had had some form of contact with the health care system within the week prior to suicide; and $9 \%$ were seen as outpatients by either a GP or psychiatrist for mental health reasons in the week prior to suicide.

\section{Discussion}

The NSMES files can serve as a rich source of information for surveillance and suicide prevention efforts. They can provide more detailed data than the provincial health service administrative databases, including as they do information on precipitating circumstances such as relationship break-ups, marital problems, employment losses, encounters with violence (either as perpetrator or victim), legal problems and problems with school, work or finances. Since NSMES conducts an autopsy on all deaths by suicide or potentially by suicide, their records necessarily include a far more complete source of medical comorbidities than any other database.

Our review of the NSMES files found that some sociodemographicinformation (i.e.age, address, sex, marital status) was recorded in all of the files, although marital status was not always consistently recorded. Other information (e.g. employment status) was not always recorded although such information would be useful for surveillance and prevention research purposes. Our findings are in keeping with the results of a 2005 study of coroners' files in England in which demographic characteristics such as sex, age and marital status were generally well recorded, but employment information was missing in over one-third of cases, precluding robust socio-economic classification. ${ }^{13}$

The relative consistency of recording basic demographic information allowed us to provide a general profile of individuals who died by suicide in Nova Scotia over a oneyear period. Our findings of higher rates among men and boys, and those in mid-life, were consistent with patterns observed in other jurisdictions. ${ }^{5,15,20,25,26,30}$ In our study, as in others, men and boys were more likely to use highly lethal means of suicide, likely increasing the odds of classification as suicide. ${ }^{15,20,25}$ Lack of consistency of collection of information regarding other risk factors prevented us from conducting more sophisticated analyses.

Information on known risk factors, such as health issues, can inform surveillance and prevention efforts. In particular, information about mental illnesses (e.g. personality disorders or major depressive episodes), substance use and/or multiple chronic physical health problems is useful since such health issues place individuals at higher risk of suicide. $^{22,31}$ However, such information was variably reported in the files we examined.
We were able to overcome this limitation, to some extent, by linking with health records from provincial administrative databases when valid encrypted health card numbers were available for decedents (101 of 108 files). Nevertheless, most health-related information was not consistently available in the NSMES files.

Firearm data may be particularly useful for informing public policy, yet our study demonstrates that most files do not contain information on ownership, license status and storage. In the case of deaths by hanging, ligature information is similarly scarce. However, this may be less relevant for policy development as ligature materials are widely available to the public. Such information may be of greater relevance in institutional settings.

Other socio-economic conditions and factors, such as living on social disability, low income, low educational attainment, significant losses (e.g. of relationships or employment), a criminal record (and related fears of arrest or imprisonment) and social isolation, are also major potential risk factors for suicide. ${ }^{32-34}$ This information is also variably recorded in the NSMES files. This problem is neither unique to Nova Scotia nor to Canada: such a limitation has also been reported by researchers examining coroners' or medical examiners' reports in other jurisdictions. ${ }^{9,13}$

There appear to be at least four main reasons for the variations across files, as well as the inconsistencies within files: (1) information is collected by different people (e.g. various investigators, police officers, etc.) who may record information to varying degrees; (2) information is collected from different sources (e.g. family, friends or physicians) who may know the decedent in different ways and to varying degrees or who may interpret the investigators' questions in different ways; (3) the medical charts of decedents may not always be requested, received or recorded consistently; and (4) there is no structured interview procedure used when most of the social and medical/health information is gathered from family and friends in particular. Collecting information from family or friends may be further complicated by their hesitation to report any declarations of intent because of financial reasons (e.g. insurance) or the stigma associated with suicide. 
TABLE 2

Demographic description of suicide cases in NSMES records from January 1,2006 , to December 31, $2006(\mathrm{~N}=108)^{\mathrm{a}}$

\begin{tabular}{lrr} 
Characteristic & \multicolumn{2}{c}{$\begin{array}{c}\text { Number of cases, } \\
\mathbf{n}(\%)\end{array}$} \\
\hline $\begin{array}{l}\text { Age, years } \\
<30\end{array}$ & 15 & $(13.9)$ \\
$30-39$ & 23 & $(21.3)$ \\
$40-49$ & 36 & $(33.3)$ \\
$50-59$ & 21 & $(19.4)$ \\
$60-69$ & 7 & $(6.5)$ \\
$\geq 70$ & 6 & $(5.6)$ \\
\hline Sex & & \\
Male & 90 & $(83.3)$ \\
Female & 18 & $(1.7)$ \\
\hline Place of Residence & & \\
Urban & 52 & $(48.1)$ \\
Rural & 56 & $(51.9)$ \\
\hline Cause of Death & & \\
$\quad$ Hanging & 42 & $(38.9)$ \\
Self-poisoning & 26 & $(24.1)$ \\
Firearm injury & 21 & $(19.4)$ \\
Drowning & 6 & $(5.6)$ \\
Blunt force injury & 5 & $(4.6)$ \\
Other & 8 & $(7.4)$ \\
\hline Location of Death & & \\
Home & 61 & $(56.5)$ \\
Public (e.g. & 30 & $(27.8)$ \\
bridge, woods) & & \\
Vehicle & 6 & $(5.6)$ \\
Property of & 6 & $(5.6)$ \\
family or friends & & \\
Other & 5 & $(4.6)$ \\
\hline
\end{tabular}

Abbreviations: NSMES, Nova Scotia Medical Examiner Service.

a All cases of death due to suicide manually extracted from NSMES records from January 1, 2006, to December 31, 2006.

These explanations all point to an overarching principle: data collection by a medical examiner or coroner takes place in an investigative context, not a research context. As such, the goal is to determine the cause and circumstances of individual deaths and, in the case of suicide, to determine intent, not to collect standardized data. However, systematically collected information would contribute to a rich source of data useful for population level surveillance and prevention research activities.

The relevance of this information is not limited to Nova Scotia. Statistics Canada has initiated a Canadian Coroner and Medical Examiner Database (CCMED) that will store information on deaths reported by coroners and medical examiners. ${ }^{35}$ This will facilitate the identification and characterization of emerging and known safety hazards, thus contributing to the prevention of avoidable deaths among Canadians. The ability of the CCMED to meet this objective will depend upon the quality and completeness of the data.

In order to provide a complete, representative Nova Scotian database, we recommend that the fields for collection be determined and populated consistently during medical examiners' investigations. While the operational impact of this change may be minimal, this endeavour is a marked conceptual departure from the way the NSMES is currently described in legislation.

\section{Limitations}

Qualitative component. Given that data were collected over a one-year period, there may be other types and sources of information provided in files outside of this period. However, many of the types and sources of data found in our review were repeated across files, suggesting that we were able to determine most, if not all, types and sources of information.

Quantitative component. We had planned to determine whether certain types of information about the suicide (e.g. information about mental health issues) might be collected systematically according to sociodemographic characteristics (e.g. age category, sex). However, during data collection and analysis we discovered that there were few structured questions consistently asked of each suicide, resulting in incomplete data capture, and therefore this type of analysis could not be undertaken. We were able to overcome this limitation in the case of health service use by linking with administrative databases, but we were otherwise unable to determine associations between variables that have been identified as risk factors for suicide (e.g. mental health problems).

Small cell sizes were also limiting. We suppressed cells smaller than 5 to prevent inadvertent identification of individuals. Future work could include preparation of a larger historical cohort.

\section{Conclusion}

To date, the data collected by NSMES in the course of its investigations have not been analysed or used for surveillance or ongoing prevention research purposes. Our study found that much of the information collected by medical examiners in Nova Scotia varies and as such cannot be fully used to develop a provincially representative, robust surveillance system inclusive of a number of suicide risk factors. There appear to be two key issues with respect to the use of medical examiners' data for suicide surveillance and prevention research: (1) inconsistencies in some of the sociodemographic information collected and recorded across files, as well as inconsistencies within the files, and (2) significant variations across files in the amount of social, medical/health and other information provided or recorded.

The use of routinely collected data provides a feasible means of surveillance. NSMES records can provide information on all deaths deemed to be due to suicide. Use of a standardized interview instrument or data collection tool in the course of investigations would help ensure completeness of the data. The instrument may include closed-ended questions, which would be useful for populating a research database; however, we recognize that the unique nature of each investigation prohibits the implementation of a single uniform set of closed-ended questions. The resulting comprehensive data set may be used to assist in our understanding of suicide in the population, including the use of common methods and associated sociodemographic factors, as well as to identify opportunities for intervention. Reconstructing the NSMES system to serve this important public health purpose will likely require legislative changes.

\section{Acknowledgements}

The authors wish to thank the Nova Scotia Department of Justice for access to the records of the Nova Scotia Medical Examiner Service (NSMES). The administrative data were made available by the Population Health Research Unit (PHRU) within Dalhousie University's Department of Community Health and Epidemiology. The Province of Nova Scotia supplies the PHRU with physician billing and hospital discharge abstracts suitable for research purposes. This study was supported by funding from the Nova Scotia Department of Health Promotion and 
Protection. The opinions, results and conclusions reported in this paper are those of the authors and are independent from the funding and data sources. No endorsement by the Nova Scotia Departments of Health and Wellness or of Justice is intended or should be inferred.

\section{References}

1. Peter Nestman; Population Health Research Unit. Suicide and attempted suicide in Nova Scotia (1995-2004): a report [Internet]. Halifax (NS): Department of Health Promotion and Protection; 2009 Feb [cited 2011 Jan 10]. Available from: http://www.gov.ns.ca/hpp /publications/Suicide_Report.pdf

2. Statistics Canada. CANSIM Table 102-4309. Mortality and potential years of life lost, by selected causes of death and sex, three-year average, Canada, provinces, territories, health regions and peer groups, occasional [Internet]. Ottawa (ON): Statistics Canada; [cited 2011 April 18]. Available from: http://www5 .statcan.gc.ca/cansim/pick-choisir?lang=e ng\&searchTypeByValue=1\&id=1024309

3. Donaldson AE, Larsen Y, Fullerton-Gleason L, Olson LM. Classifying undetermined poisoning deaths. Inj Prev. 2006;12:338-43.

4. Parai J, Kreiger N, Tomlinson G, Adlaf E. The validity of the certification of manner of death by Ontario coroners. Ann Epidemiol. 2006;16:805-11.

5. Health Canada. A report on mental illnesses in Canada. Ottawa (ON): Health Canada; 2002 Oct [cited 2010 Jul 13]. Available from: http://www.phac-aspc.gc.ca/publicat /miic-mmac/pdf/men_ill_e.pdf

6. Government of Nova Scotia. Office of the Chief Medical Examiner. Halifax (NS): Government of Nova Scotia; [updated 2009 Apr 4; cited 2010 Jul 13]. Available from: http://www.gov.ns.ca/just/CME.asp

7. Government of Nova Scotia. Fatality investigations act, 2001. Halifax (NS): Government of Nova Scotia; 2002 [cited 2010 Jul 13]. Available from: http://www.gov.ns.ca /legislature/legc/statutes/fatalinv.htm
8. Moscicki EK. North American perspectives: epidemiology of suicide. Int Psychogeriatr. 1995;7:137-48.

9. Parrish G. Assessing and improving the quality of data from medical examiners and coroners [Internet]. Atlanta (GA): Centres for Disease Control and Prevention; 1995 [cited 2010 Jul 13]. Available at: http://www.cdc .gov/nchs/data/ice/ice95v1/C25.pdf

10. Isaacs S, Keogh S, Menard C, Hockin J. Suicide in the Northwest Territories: a descriptive review. Chronic Dis Can 1998; 19:152-6.

11. Poulin C, Stein J, Butt J. Surveillance of drug overdose deaths using medical examiner data. Chronic Dis Can. 1998;19:177-82.

12. Snowdon J, Baume P. A study of suicides of older people in Sydney. Int $\mathrm{J}$ Geriatr Psychiatry. 2002;17:261-9.

13. Bennewith O, Hawton K, Simkin S, Sutton L, Kapur N, Turnbull P, et al. The usefulness of coroners' data on suicides for providing information relevant to prevention. Suicide Life Threat Behav. 2005;35:607-14.

14. Comstock RD, Mallonee S, Jordan F. A comparison of two surveillance systems for deaths related to violent injury. Inj Prev. 2005;111:58-63.

15. Shields LB, Hunsaker DM, Hunsaker JC $3^{\text {rd }}$. Suicide: a ten-year retrospective review of Kentucky medical examiner cases. J Forensic Sci. 2005 May;50(3):613-7.

16. Juurlink DN, Mamdani MM, Kopp A, Redelmeier DA. The risk of suicide with selective serotonin reuptake inhibitors in the elderly. Am J Psychiatry. 2006;163:813-21.

17. Sutton L, Hawton K, Simkin S, Turnbull P, Kapur N, Bennewith O, et al. Gunshot suicides in England--a multicentre study based on coroners' records. Soc Psychiatry Psychiatr Epidemiol. 2005 Apr;40:324-8.

18. Hempstead K. The geography of self-injury: spatial patterns in attempted and completed suicide. Soc Sci Med. 2006;62:3186-96.
19. Paulozzi LJ, Mercy J, Frazier L Jr, Annest JL; Centers for Disease Control and Prevention. CDC's National Violent Death Reporting System: background and methodology. Inj Prev. 2004;10:47-52.

20. Sanford C, Marshall SW, Martin SL, CoyneBeasley T, Waller AE, Cook PJ, et al. Deaths from violence in North Carolina, 2004: how deaths differ in females and males. Inj Prev. 2006 Dec;12 Suppl 2:ii10-ii16.

21. Canadian Red Cross. The facts about drowning in Canada: 2005 edition [Internet]. Ottawa (ON): Canadian Red Cross; 2005 [cited 2010 Oct 20]. Available at: http:// www.croixrouge.ca/cmslib/general /drowningreport2005_en.pdf

22. Cheng AT, Chen TH, Chen CC, Jenkins R. Psychosocial and psychiatric risk factors for suicide. Case-control psychological autopsy study. Br J Psychiatry. 2000;177:360-5.

23. Séguin M, Lesage A, Turecki G, Daigle F, Guy A. Research project on deaths by suicide in New Brunswick between April 2002 and May 2003 [Internet]. Montreal (QC): Douglas Mental Health University Institute; 2005 Apr [cited 2010 Jul 13]. Available at: http:// www.gnb.ca/0055/pdf/3182-e.pdf

24. Garlow SJ. Age, gender, and ethnicity differences in patterns of cocaine and ethanol use preceding suicide. Am J Psychiatry. 2002 Apr;159:615-9.

25. Langlois S, Morrison P. Suicide deaths and suicide attempts. Health Rep. 2002;13(2): 9-22.

26. Paris J. Predicting and preventing suicide: do we know enough to do either? Harv Rev Psychiatry. 2006;14:233-40.

27. Shields LB, Hunsaker DM, Hunsaker JC $3^{\text {rd }}$. Adolescent and young adult suicide: a 10-year retrospective review of Kentucky Medical Examiner cases. J Forensic Sci. 2006 Jul;51 (4): 874-9.

28. Rowe JL, Bruce ML, Conwell Y. Correlates of suicide among home health care utilizers who died by suicide and community controls. Suicide Life Threat Behav. 2006 Feb;36(1): 65-75. 
29. Statistics Canada. 2006 Community Profiles: Halifax, Nova Scotia (Code1209034) (table) [Internet]. Ottawa (ON): Statistics Canada; 2007 [cited 2011 Jan 6]. [Statistics Canada, Catalogue No.: 92-591-XWE]. Available at: http:// www12.statcan.ca/census-recensement /2006/dp-pd/prof/92-591/index.

cfm?Lang $=\mathrm{E}$

30. Hu G, Wilcox HC, Wissow L, Baker SP. Midlife suicide: an increasing problem in U.S. whites, 1999-2005. Am J Prev Med. 2008; 35:589-93.

31. Qin P, Agerbo E, Mortensen PB. Suicide risk in relation to socioeconomic, demographic, psychiatric, and familial factors: a national register-based study of all suicides in Denmark, 1981-1997. Am J Psychiatry. 2003; 160:765-72.

32. Chan WS, Yip PS, Wong PW, Chen EY. Suicide and unemployment: what are the missing links? Arch Suicide Res. 2007;11: 327-35.

33. Rush B, Koegl CJ. Prevalence and profile of people with co-occurring mental and substance use disorders within a comprehensive mental health system. Can J Psychiatry. 2008;53:810-21.

34. Stack S, Wasserman I. Economic strain and suicide risk: a qualitative analysis. Suicide Life Threat Behav. 2007;37:103-12.

35. Statistics Canada. Canadian Coroner and Medical Examiner Database (CCMED). Ottawa (ON): Statistics Canada; [modified 2009 Dec 16; cited 2010 Jul 13]. Available at: http://www.statcan.gc.ca/cgi-bin/imdb /p2SV.pl?Function $=$ getSurvey \&SDDS $=5125 \&$ lang $=$ en $\& \mathrm{db}=\mathrm{imdb} \& \mathrm{adm}=8 \& \mathrm{dis}=2$ 